\title{
When object production tunes the efficiency of membrane systems
}

\author{
David Orellana-Martín, Miguel Á. Martínez-del-Amor *, Ignacio Pérez-Hurtado, \\ Agustín Riscos-Núñez, Luis Valencia-Cabrera, Mario J. Pérez-Jiménez
}

Research Group on Natural Computing, Department of Computer Science and Artificial Intelligence, Universidad de Sevilla, Avda. Reina Mercedes s/n, 41012, Sevilla, Spain

Keywords:

Membrane Computing

Active membranes

Minimal cooperation

Computational complexity

\begin{abstract}
A B S T R A C T
P systems with active membranes is one of the most studied models within the field of Membrane Computing. Simulating the organization and behavior of the living cells through a tree-like structure and abstracting the mechanisms that help the cell to keep alive into rules (evolution, communication, dissolution and division rules), they have been used to solve several computationally hard problems. We are dealing with non-cooperative systems here, that is, the number of reactives in a rule is always one. Even then, it has been proven that problems from the class PSPACE can be solved, so in order to acquire a minimal model that can solve computationally hard problems, polarizations are removed. In this paper we find the relevance of the length of the right-hand side of the rule, being necessary when using separation rules and being irrelevant when division rules are used, improving some solutions previously presented, restricting the right-hand side of the rules, obtaining new frontiers of efficiency in this framework. The state of the art of these systems is presented in a graphical way.
\end{abstract}

\section{Introduction}

Membrane Computing, first introduced in [15], is a computational paradigm inspired by the structure and behavior of living cells. Here, chemical elements flow through intra-cellular membranes, interchanging information between different compartments of the cell or between cells when they communicate within living tissues. Chemical reactions occur throughout the cell, taking into account both the reactants and the environment they are in, and producing new elements, even changing the structure of the cell itself. This behavior is seen in one of the most important processes of the cell, mitosis, where the cell ends dividing into two new identical cells with the same genetic material of the original one.

In [5], P systems with active membranes are presented, introducing division rules inspired by the mitosis mechanism. At first, these systems were non-cooperative by means of objects, that is, rules were limited to use only one object as a reactive. Even with this restriction, computationally hard problems could be solved in polynomial time, so polarization were removed in order to obtain new frontiers of efficiency. In the framework of polarizationless $\mathrm{P}$ systems, forbidding or allowing

\footnotetext{
* Corresponding author.

E-mail addresses: dorellana@us.es (D. Orellana-Martín), mdelamor@us.es (M.Á. Martínez-del-Amor), perezh@us.es (I. Pérez-Hurtado), ariscosn@us.es (A. Riscos-Núñez), lvalencia@us.es (L. Valencia-Cabrera), marper@us.es (M.J. Pérez-Jiménez).
} 
dissolution rules seems the key to pass from the non-efficiency to the efficiency [3]. In [14], the abstraction of membrane fission is introduced in the active membranes framework, giving way to separation rules.

In [20], minimal cooperation is introduced in the framework of polarizationless P systems with active membranes, inspired by the interaction of two chemical elements in a single reaction, and later on, cooperation is used in communication rules instead of object evolution rules [22,23] obtaining polynomial-time solutions to the SAT problem by means of polarizationless P systems using minimal cooperation and minimal production in communication rules, whether they are send-in or send-out, simple object evolution rules and division rules for elementary and non-elementary membranes. In this work we provide a new frontier of efficiency based on the use of separation rules instead of division rules, and providing a graphical way to see the current computational complexity landscape in these kinds of systems.

The paper is organized as follows. Next section briefly describes some preliminaries in order to make the work selfcontained. In Section 3, syntax and semantics of polarizationless P systems with active membranes by using membrane division rules or membrane separation rules are introduced. Definition of Recognizer membrane systems is recalled in Section 4, as a framework to provide efficient solutions to decision problems. The concept of bounded minimal cooperation in object evolution and in communication rules is explained in sections 5 and 6. Next section is devoted to describe the limits of polarizationless P systems that make use of separation rules and minimal cooperation and minimal production in communication rules. In Section 8 we improve the solution from [1] to solve QSAT problem using simple evolution rules instead of classical ones. An overview of P systems with active membranes both using and not using cooperation is given in Section 9, with some figures lightening the current landscape of the study of computational complexity.

The paper ends with some open problems and concluding remarks.

\section{Preliminaries}

An alphabet $\Gamma$ is a non-empty set and their elements are called symbols. A string $u$ over $\Gamma$ is an ordered finite sequence of symbols, that is, a mapping from a natural number $n \in \mathbb{N}$ onto $\Gamma$. The number $n$ is called the length of the string $u$ and it is denoted by $|u|$, that is, the length of a string is the number of occurrences of symbols that it contains. The empty string (with length 0 ) is denoted by $\lambda$. The set of all strings over an alphabet $\Gamma$ is denoted by $\Gamma^{*}$. A language over $\Gamma$ is a subset of $\Gamma^{*}$.

A multiset over an alphabet $\Gamma$ is an ordered pair $(\Gamma, f)$ where $f$ is a mapping from $\Gamma$ onto the set of natural numbers $\mathbb{N}$. The support of a multiset $m=(\Gamma, f)$ is defined as $\operatorname{supp}(m)=\{x \in \Gamma \mid f(x)>0\}$. A multiset is finite (respectively, empty) if its support is a finite (respectively, empty) set. We denote by $\emptyset$ the empty multiset and we denote by $M_{f}(\Gamma)$ the set of all finite multisets over $\Gamma$.

Let $m_{1}=\left(\Gamma, f_{1}\right), m_{2}=\left(\Gamma, f_{2}\right)$ be multisets over $\Gamma$, then the union of $m_{1}$ and $m_{2}$, denoted by $m_{1}+m_{2}$, is the multiset ( $\Gamma, g)$, where $g(x)=f_{1}(x)+f_{2}(x)$ for each $x \in \Gamma$. We say that $m_{1}$ is contained in $m_{2}$ and we denote it by $m_{1} \subseteq m_{2}$, if $f_{1}(x) \leq f_{2}(x)$ for each $x \in \Gamma$. The relative complement of $m_{2}$ in $m_{1}$, denoted by $m_{1} \backslash m_{2}$, is the multiset $(\Gamma, g)$, where $g(x)=f_{1}(x)-f_{2}(x)$ if $f_{1}(x) \geq f_{2}(x)$, and $g(x)=0$ otherwise.

Let us recall that a free tree (tree, for short) is a connected, acyclic, undirected graph. A rooted tree is a tree in which one of the vertices (called the root of the tree) is distinguished from the others. In a rooted tree the concepts of ascendants and descendants are defined in a usual way. Given a node $x$ (different from the root), if the last edge on the (unique) path from the root of the tree to the node $x$ is $\{x, y\}$ (in this case, $x \neq y$ ), then $y$ is the parent of node $x$ and $x$ is a child of node $y$. The root is the only node in the tree with no parent. A node with no children is called a leaf (see [2] for details).

\section{Polarizationless P systems with active membranes}

Let us briefly recall some definitions of P systems models that will be used in the paper (see [7] for details).

A basic transition P system is a membrane system whose rules are of the following forms: evolution, communication, and dissolution. In these systems the size of the membrane structure does not increase, but an exponential workspace (in terms of number of objects) can be constructed in linear time, e.g., via evolution rules of the type $\left[a \rightarrow a^{2}\right]_{h}$. Nevertheless, such capability is not enough to efficiently solve NP-complete problems, unless $\mathbf{P}=\mathbf{N P}$ (see [4] for details).

Replication is one of the most important functions of a cell and, in ideal circumstances, a cell produces two identical copies by division. Bearing in mind that the reactions which take place in a cell are related to membranes, division rules for elementary and non-elementary membranes are considered in the so-called $P$ systems with active membranes. Such variant was first introduced by Gh. Păun [6] and it has associated electrical charges with membranes but the rules are non-cooperative and there are not priorities. Nevertheless, the class of all problems solvable in polynomial time and in a uniform way by means of families of $\mathrm{P}$ systems with active membranes which use division for elementary and nonelementary membranes contains class PSPACE and it is contained in class EXP [12]. Thus, in order to provide efficient solutions to computationally hard problems, this framework seems to be too powerful from the computational complexity point of view.

In this paper, electrical charges are removed from $P$ systems with active membranes. In the figures, we refer to membrane systems that make use of polarizations, but we do not explain them widely because they are not used here. Readers that want to know more about these systems can take a look at [5]. Two different ways of producing an exponential number of 
membranes in linear time will be considered: division and separation rules (abstractions of mitosis and membrane fission processes, respectively).

\subsection{Polarizationless $P$ systems with active membranes: syntax}

Definition 3.1. A polarizationless $\mathrm{P}$ system with active membranes and membrane division of degree $q \geq 2$ is a tuple $\left(\Gamma, H, \mu, \mathcal{M}_{1}, \ldots, \mathcal{M}_{q}, \mathcal{R}, i_{\text {out }}\right)$, where:

- $\Gamma$ is a finite alphabet whose elements are called objects;

- $H$ is a finite alphabet such that $H \cap \Gamma=\emptyset$ whose elements are called labels;

- $\mu$ is a labeled rooted tree consisting of $q$ nodes injectively labeled by elements of $H$ (the leaves of $\mu$ are called elementary membranes and we denote by $i_{\text {skin }}$ the label of the root of $\mu$ );

- $\mathcal{M}_{1}, \ldots, \mathcal{M}_{q}$ are multisets over $\Gamma$;

- $\mathcal{R}$ is a finite set of rules, of the following forms:

$\left(a_{0}\right)[a \rightarrow u]_{h}$, for $h \in H, a \in \Gamma, u \in M_{f}(\Gamma)$ (object evolution).

$\left(b_{0}\right) a[]_{h} \rightarrow[b]_{h}$, for $h \in H \backslash\left\{i_{\text {skin }}\right\}, a, b \in \Gamma$ (send-in communication).

$\left(c_{0}\right)[a]_{h} \rightarrow b[]_{h}$, for $h \in H, a, b \in \Gamma$ (send-out communication).

$\left(d_{0}\right)[a]_{h} \rightarrow b$, for $h \in H \backslash\left\{i_{\text {out }}, i_{\text {skin }}\right\}, a, b \in \Gamma$ (dissolution).

$\left(e_{0}\right)[a]_{h} \rightarrow[b]_{h}[c]_{h}$, for $h \in H \backslash\left\{i_{\text {out }}, i_{\text {skin }}\right\}, h$ is the label of an elementary membrane, $a, b, c \in \Gamma$ (division for elementary membranes).

$\left(f_{0}\right)\left[[]_{h_{0}}[]_{h_{1}}\right]_{h} \rightarrow\left[[]_{h_{0}}\right]_{h}\left[[]_{h_{1}}\right]_{h}$, where $h \in H \backslash\left\{i_{\text {out }}, i_{\text {skin }}\right\}$ and $h_{0}, h_{1} \in H$ (division for non-elementary membranes).

- $i_{\text {out }} \in H \cup\{e n v\}$, where env $\notin H$, and in the case $i_{\text {out }} \in H$, $i_{\text {out }}$ is the label of a leaf of $\mu$.

Definition 3.2. A polarizationless $\mathrm{P}$ system with active membranes and membrane separation of degree $q \geq 2$ is a tuple $\left(\Gamma, \Gamma_{0}, \Gamma_{1}, H, \mu, \mathcal{M}_{1}, \ldots, \mathcal{M}_{q}, \mathcal{R}, i_{\text {out }}\right)$, where:

- $\left(\Gamma, H, \mu, \mathcal{M}_{1}, \ldots, \mathcal{M}_{q}, i_{\text {out }}\right)$ is as the previous Definition.

- $\left\{\Gamma_{0}, \Gamma_{1}\right\}$ is a partition of $\Gamma$ and $\left\{H_{0}, H_{1}\right\}$ is a partition of $H$;

- $\mathcal{R}$ is a finite set of rules, of the following forms:

$\left(a_{0}\right)[a \rightarrow u]_{h}$, for $h \in H, a \in \Gamma, u \in M_{f}(\Gamma)$ (object evolution).

$\left(b_{0}\right) a[]_{h} \rightarrow[b]_{h}$, for $h \in H, a, b \in \Gamma$ and $h \in H \backslash\left\{i_{\text {skin }}\right\}$ (send-in communication).

$\left(c_{0}\right)[a]_{h} \rightarrow b[]_{h}$, for $h \in H, a, b \in \Gamma$ (send-out communication).

$\left(d_{0}\right)[a]_{h} \rightarrow b$, for $h \in H \backslash\left\{i_{\text {out }}, i_{\text {skin }}\right\}, a, b \in \Gamma$ (dissolution).

$\left(e_{0}\right)[a]_{h} \rightarrow\left[\Gamma_{0}\right]_{h}\left[\Gamma_{1}\right]_{h}$, for $h \in H \backslash\left\{i_{\text {out }}, i_{\text {skin }}\right\}, h$ is the label of an elementary membrane, $a \in \Gamma$ (separation)

$\left(f_{0}\right)\left[[]_{h_{0}}[]_{h_{1}}\right]_{h} \rightarrow\left[\Gamma_{0}[]_{h_{0}}\right]_{h}\left[\Gamma_{1}[]_{h_{1}}\right]_{h}$, where $h \in H \backslash\left\{i_{\text {out }}, i_{\text {skin }}\right\}, h_{0} \in H_{0}$ and $h_{1} \in H_{1}$ (separation rules for nonelementary membranes).

A polarizationless $\mathrm{P}$ system with active membranes of degree $q \geq 2$, can be viewed as a set of $q$ membranes, labeled by elements of $H$, arranged in a hierarchical structure $\mu$ given by a rooted tree (called membrane structure) whose root is called the skin membrane, such that: (a) $\mathcal{M}_{1}, \ldots, \mathcal{M}_{q}$ represent the finite multisets of objects initially placed in the $q$ membranes of the system; (b) $\mathcal{R}$ is a finite set of rules over $\Gamma$ associated with the labels; and (c) $i_{\text {out }} \in H \cup\{$ env $\}$ indicates the output region. We use the term region $i$ to refer to membrane $i$ in the case $i \in H$ and to refer to the "environment" of the system in the case $i=e n v$. In these kinds of $\mathrm{P}$ systems there are mechanisms, implemented by division rules or separations rules, able to generate an exponential workspace (in terms of number of membranes and objects) in polynomial time. This allows us to describe brute force algorithms in these systems.

\subsection{Polarizationless $P$ systems with active membranes: semantics}

An instantaneous description or a configuration $\mathcal{C}_{t}$ at an instant $t$ of a polarizationless $\mathrm{P}$ system with active membranes is described by the following elements: (a) the membrane structure at instant $t$, and (b) all multisets of objects over $\Gamma$ associated with all the membranes present in the system at that moment.

An object evolution rule $[a \rightarrow u]_{h}$ for $h \in H, a \in \Gamma, u \in M_{f}(\Gamma)$ is applicable to a configuration $\mathcal{C}_{t}$ at an instant $t$, if there exists a membrane labeled by $h$ in $\mathcal{C}_{t}$ which contains object $a$. When applying such a rule, object $a$ is consumed and objects from multiset $u$ are produced in that membrane.

A send-in communication rule $a[]_{h} \rightarrow[b]_{h}$ for $h \in H, a, b \in \Gamma$ is applicable to a configuration $\mathcal{C}_{t}$ at an instant $t$, if there exists a membrane labeled by $h$ in $\mathcal{C}_{t}$ such that $h$ is not the label of the root of $\mu$ and its parent membrane contains object $a$. When applying such a rule, object $a$ is consumed from the parent membrane and object $b$ is produced in the corresponding membrane $h$.

A send-out communication rule $[a]_{h} \rightarrow b$ [ $]_{h}$ for $h \in H, a, b \in \Gamma$ is applicable to a configuration $\mathcal{C}_{t}$ at an instant $t$, if there exists a membrane labeled by $h$ in $\mathcal{C}_{t}$ such that it contains object $a$. When applying such a rule, object $a$ is consumed from such membrane $h$ and object $b$ is produced in the parent of such membrane. 
A dissolution rule $[a]_{h} \rightarrow b$ for $h \in H \backslash\left\{i_{\text {out }}\right\}, a, b \in \Gamma$ is applicable to a configuration $\mathcal{C}_{t}$ at an instant $t$, if there exists a membrane labeled by $h$ in $\mathcal{C}_{t}$, different from the skin membrane and the output region, such that it contains object $a$. When applying such a rule, object $a$ is consumed, membrane $h$ is dissolved and its objects are sent to the parent (or the first ancestor that has not been dissolved).

A division rule $[a]_{h} \rightarrow[b]_{h}[c]_{h}$ for $h \in H \backslash\left\{i_{\text {out }}\right\}, a, b, c \in \Gamma$, is applicable to a configuration $\mathcal{C}_{t}$ at an instant $t$, if there exists an elementary membrane labeled by $h$ in $\mathcal{C}_{t}$, different from the skin membrane and the output region, such that it contains object $a$. When applying a division rule $[a]_{h} \rightarrow[b]_{h}[c]_{h}$ to a membrane labeled by $h$ in a configuration $\mathcal{C}_{t}$, under the influence of object $a$, the membrane with label $h$ is divided into two membranes with the same label; in the first copy, object $a$ is replaced by object $b$, in the second one, object $a$ is replaced by object $c$; all the other objects are replicated and copies of them are placed in the two new membranes.

A division rule [[ $\left.]_{h_{0}}[]_{h_{1}}\right]_{h} \rightarrow\left[[]_{h_{0}}\right]_{h}$ [[ $\left.]_{h_{1}}\right]_{h}$ is applicable to a configuration $\mathcal{C}_{t}$ at an instant $t$, if there exists a membrane labeled by $h$ in $\mathcal{C}_{t}$, different from the skin membrane and the output region, which contains a membrane labeled by $h_{0}$ and another membrane labeled by $h_{1}$. When applying such a division rule to a membrane labeled by $h$ in a configuration $\mathcal{C}_{t}$, the membrane with label $h$ is divided into two membranes with the same label; the first copy inherits membrane $h_{0}$ with its contents, and the second copy inherits membrane $h_{1}$ with its contents. Besides, if the membrane labeled by $h$ contains more membranes other than those with the labels $h_{0}, h_{1}$, then such membranes are duplicated so that they become part of the contents of both new copies of the membrane $h$.

A separation rule $[a]_{h} \rightarrow\left[\Gamma_{0}\right]_{h}\left[\Gamma_{1}\right]_{h}$ for $h \in H, a \in \Gamma$, is applicable to a configuration $\mathcal{C}_{t}$ at an instant $t$, if there exists an elementary membrane labeled by $h$ in $\mathcal{C}_{t}$, different from the skin membrane and the output region, such that it contains object $a$. When applying such a rule, the membrane is separated into two membranes with the same label; at the same time, object $a$ is consumed and the multiset of objects contained in membrane $h$ gets distributed: the objects from $\Gamma_{0}$ are placed in the first membrane, those from $\Gamma_{1}$ are placed in the second membrane.

A separation rule $\left[[]_{h_{0}}[]_{h_{1}}\right]_{h} \rightarrow\left[\Gamma_{0}[]_{h_{0}}\right]_{h}\left[\Gamma_{1}[]_{h_{1}}\right]_{h}$, where $h, h_{0}, h_{1}$ are labels such that $h_{0} \in H_{0}$ and $h_{1} \in H_{1}$, is applicable to a configuration $\mathcal{C}_{t}$ at an instant $t$, if there exists a membrane labeled by $h$ in $\mathcal{C}_{t}$, different from the skin membrane and the output region, such that it contains a membrane labeled by $h_{0}$ and another membrane labeled by $h_{1}$. When applying such a separation rule to a membrane labeled by $h$ in a configuration $\mathcal{C}_{t}$, that membrane is separated into two membranes with the same label, in such a way that the contents (multiset of objects and inner membranes) are distributed as follows: The first membrane receives the multiset of objects from $\Gamma_{0}$, and all inner membranes whose label belongs to $H_{0}$; and the second membrane receives the multiset of objects from $\Gamma_{1}$, and all inner membranes whose label belongs to $H_{1}$.

In polarizationless $\mathrm{P}$ systems with active membranes, the rules are applied according to the following principles:

- The rules associated with membranes labeled with $h$ are used for all copies of this membrane.

- At one transition step, one object can be used by only one rule (chosen in a non-deterministic way).

- At one transition step, a membrane can be the subject of only one rule of types $\left(b_{0}\right)-\left(f_{0}\right)$, and then it is applied at most once.

- Object evolution rules can be simultaneously applied to a membrane with one rule of types $\left(b_{0}\right)-\left(f_{0}\right)$. Object evolution rules are applied in a maximally parallel manner.

- If at the same time a membrane labeled with $h$ is divided by a rule of type $\left(e_{0}\right)$ or $\left(f_{0}\right)$ and there are objects in this membrane which evolve by means of rules of type $\left(a_{0}\right)$, then we suppose that first the evolution rules of type $\left(a_{0}\right)$ are used, changing the objects, and then the division (or the separation) is produced. Of course, this process takes only one transition step.

- The skin membrane and the output membrane can never get divided, separated, nor dissolved.

Let us notice that in these kinds of $\mathrm{P}$ systems the environment plays a passive role in the following sense: along any computation, the environment only can receive objects from the system but it cannot send objects into the system.

\section{Polynomial complexity classes of recognizer membrane systems}

In what follows, a membrane system denotes a P system of any of the different variants considered in the previous section. The concept of recognizer membrane system is defined as usual (see [9] for details). It is worth noting that in these systems the working alphabet $\Gamma$ has two distinguished objects yes and no, there exists an input alphabet $\Sigma$, the initial multisets of the system are multisets over $\Gamma \backslash \Sigma$, the output region is the environment, all computations halt, and for every computation, then either object yes or object no (but not both) must have been released into the environment, and only at the last step of the computation.

If $\Pi$ is a recognizer membrane system then, for each multiset $m$ over the input alphabet $\Sigma$, we denote by $\Pi+m$ the system $\Pi$ where at the initial configuration, the multiset $m$ has been added to the input membrane. Thus, we have an initial configuration associated with each input multiset $m$ over $\Sigma$ in this kind of systems. Any computation of $\Pi+m$ starts from such an initial configuration. 
We denote by $\mathcal{D} \mathcal{A} \mathcal{M}^{0}(\alpha, \beta, \delta, \gamma)$ where $\alpha \in\{-e,+e\}, \beta \in\{-c,+c\}, \delta \in\{-d,+d\}$ and $\gamma \in\{-n,+n\}$, the class of all recognizer polarizationless $\mathrm{P}$ systems with active membranes and division rules. The meaning of parameters $\alpha, \beta, \delta$ and $\gamma$ is the following:

- if $\alpha=+e$ (resp., $\alpha=-e$ ) then evolution rules are permitted (resp., forbidden).

- if $\beta=+c$ (resp., $\beta=+c$ ) then communication rules are permitted (resp., forbidden).

- if $\delta=+d$ (resp., $\delta=-d$ ) then dissolution rules are permitted (resp., forbidden).

- if $\gamma=+n$ (resp., $\gamma=-n$ ) then division rules for elementary and non-elementary membranes are permitted (resp., only division rules for elementary membranes are permitted).

In a similar way, notation $\mathcal{S A M}^{0}(\alpha, \beta, \delta, \gamma)$ is considered when we use separation rules instead of division rules. Next, let us recall the concept of efficient solvability by means of a family of recognizer membrane systems (see [8] for more details).

Definition 4.1. Let $\mathcal{R}$ be a class of recognizer membrane systems. We say that a decision problem $X$ is solvable in polynomial time by a family $\Pi=\{\Pi(n) \mid n \in \mathbb{N}\}$ of systems from $\mathcal{R}$, in a uniform way, denoted by $X \in$ PMC $_{\mathcal{R}}$, if the following hold:

- the family $\Pi$ is polynomially uniform by Turing machines;

- there exists a pair (cod,s) of polynomial-time computable functions over $I_{X}$ such that:

- for each instance $u \in I_{X}, s(u)$ is a natural number and $\operatorname{cod}(u)$ is an input multiset of the system $\Pi(s(u))$;

- for each $n \in \mathbb{N}, s^{-1}(n)$ is a finite set;

- the family $\Pi$ is polynomially bounded, sound and complete with regard to $(X, \operatorname{cod}, s)$

The polynomial complexity class $\mathbf{P} \mathbf{M} \mathbf{C}_{\mathcal{R}}$ is closed under polynomial-time reduction and under complement [10].

\section{Minimal cooperation in object evolution rules of polarizationless $P$ systems with active membranes}

Let us recall that polarizationless $\mathrm{P}$ systems with active membranes are non-cooperative systems, that is, the left-hand side of the rules of these systems has only one object (the objects do not directly interact). Moreover, with the exception of object evolution rules, single objects are always transformed into single objects (the two objects produced by a division rule are placed in two different compartments/membranes).

Let us also recall that by using the dependency graph technique it has been shown that if dissolution rules are forbidden, then only tractable problems can be solved in an efficient way by families of polarizationless $\mathrm{P}$ systems with active membranes even using division rules for non-elementary membranes (see [3] for details), that is, $\mathbf{P M C}_{\mathcal{D} \mathcal{A M}}{ }^{(+e,+c-d,+n)}=\mathbf{P}$. It is worth pointing out that in polarizationless $\mathrm{P}$ systems with active membranes and without dissolution rules, the term "non-elementary membrane" is static, that is, in any configuration of any computation of the system, a membrane is elementary if and only if it is elementary at the initial configuration of the system (a "non-elementary membrane" never can "evolve" to an "elementary membrane").

Cooperation rules take a relevant role in the efficiency of polarizationless $P$ systems with active membranes since it gives to the systems enough power to solve hard problems efficiently. Some variants have been studied:

- Minimal cooperation (mc): object-evolution rules are of the form $[u \rightarrow v]_{h}$, where $u, v \in M(\Gamma)$ and $1 \leq|u| \leq 2$.

- Primary minimal cooperation (pmc): object-evolution rules of the form $[u \rightarrow v]_{h}$, where $u, v \in M(\Gamma)$ and $1 \leq$ $|u|,|v| \leq 2$.

- Bounded minimal cooperation (bmc): object-evolution rules of the form $[u \rightarrow v]_{h}$, where $u, v \in M(\Gamma)$ and $1 \leq|u| \leq$ $|v| \leq 2$.

- Minimal cooperation and minimal production (mcmp): object-evolution rules of the form $[u \rightarrow a]_{h}$, where $u \in M(\Gamma)$, $a \in \Gamma$ and $1 \leq|u| \leq 2$.

We have to explore another kind of evolution rules, but without cooperation. They are called simple evolution rules, indicated as $+e_{s}$, and are of the form $[a \rightarrow b]_{h}$, where $a, b \in \Gamma$ and $h \in H$.

We denote by $\mathcal{D} \mathcal{A} \mathcal{M}^{0}(\alpha, \beta, \gamma, \delta)$ (resp., $\mathcal{S} \mathcal{A} \mathcal{M}^{0}(\alpha, \beta, \gamma, \delta)$ ) the class of all recognizer polarizationless $\mathrm{P}$ systems with active membranes, cooperation in object evolution rules and division (resp., separation) rules, letting $\alpha \in$ $\{m c, p m c, b m c, m c m p\}$, or using simple evolution rules if $\alpha=+e_{s}$.

Let us notice that standard notation in the literature referring to polarizationless $\mathrm{P}$ systems with active membranes $\left(\mathcal{A} \mathcal{M}^{0}(\gamma, \delta)\right)$ corresponds, within this new notation, to the class $\mathcal{D} \mathcal{A} \mathcal{M}^{0}(\alpha, \beta, \gamma, \delta)$.

\section{Minimal cooperation in communication rules of polarizationless $P$ systems with active membranes}

In terms of efficiency, it seems that cooperation plays an active role when used in object evolution rules, so it seems clear that we could try to consider this behavior in other kind of rules. Classical communication rules take an object from a 
membrane to another one (to the parent membrane if it is a send-in communication rule, and to a child membrane if it is a send-out communication rule).

Membranes could act as a cooperator that takes two objects from a membrane and "transforms" them into a new one. Then, we can define some variants:

- Minimal cooperation and minimal production in send-in communication rules ( $\left.\mathbf{m c m p}_{\text {in }}\right)$ : send-in communication rules are of the form $u[]_{h} \rightarrow[b]_{h}$, where $u \in M(\Gamma), b \in \Gamma, 1 \leq|u| \leq 2$ and send-in rules remain $[a]_{h} \rightarrow b[]_{h}$.

- Minimal cooperation and minimal production in send-out communication rules $\left(\mathbf{m c m p}_{\text {out }}\right)$ : send-out communication rules are of the form $[u]_{h} \rightarrow a[]_{h}$, where $u \in M(\Gamma), a \in \Gamma, 1 \leq|u| \leq 2$ and send-in rules remain $a[]_{h} \rightarrow[b]_{h}$.

- Minimal cooperation and minimal production in both send-in and send-out rules $\left(\mathbf{m c m p}_{\text {in-out }}\right)$ : send-in communication rules are of the form $u[]_{h} \rightarrow[b]_{h}$, where $u \in M(\Gamma), b \in \Gamma, 1 \leq|u| \leq 2$ and send-out communication rules are of the form $[u]_{h} \rightarrow a[]_{h}$, where $u \in M(\Gamma), a \in \Gamma$ and $1 \leq|u| \leq 2$

\section{Limits on efficient computations in $\mathcal{S} \mathcal{A} \mathcal{M}^{0}\left(+e_{s}, m c m p_{\text {in-out }},+d,+n\right)$}

In this section we study the computational efficiency of polarizationless $\mathrm{P}$ systems with active membranes, dissolution rules and minimal cooperation in communication rules when separation rules (for elementary and non-elementary membranes) are considered as a mechanism to generate an exponential workspace in linear time. Specifically, we will show that these kinds of $\mathbf{P}$ systems can only solve problems in class $\mathbf{P}$ in an efficient way. The proof is inspired on a similar result, obtained in the framework of polarizationless P systems with active membranes and minimal cooperation in object evolution rules [19].

Let $\Pi=\left(\Gamma, \Gamma_{0}, \Gamma_{1}, \Sigma, H, H_{0}, H_{1}, \mu, \mathcal{M}_{1}, \ldots, \mathcal{M}_{q}, \mathcal{R}, i_{\text {in }}, i_{\text {out }}\right)$ be a recognizer P system from $\mathcal{S A M}^{0}\left(+e_{s}, m c m p p_{\text {in }}\right.$ out, $+d,+n)$. In what follows we use the concepts of notations from [11].

- We denote by $p(i)$ (resp., ch(i)) the label of the parent (resp., a child) of the membrane labeled by $i$, the parent of the skin membrane is the environment (we write $p(1)=0$ ). We denote by $\mathcal{R}_{E}$ (resp., $\mathcal{R}_{C}, \mathcal{R}_{D}$ and $\mathcal{R}_{S}$ ) the set of evolution rules (resp., communication, dissolution and separation rules) of $\Pi$. We will fix total orders in $\mathcal{R}_{E}, \mathcal{R}_{C}, \mathcal{R}_{D}$ and $\mathcal{R}_{S}$.

- Let $\mathcal{C}$ be a computation of $\Pi$, and $\mathcal{C}_{t}$ an arbitrary configuration of $\mathcal{C}$. With respect to the number of objects of the system, let us notice that by applying a single rule, this number remains unchanged or decreases by one. Thus, the total number of objects in $\mathcal{C}_{t}$ is, at most, $M$, being $M=\left|\mathcal{M}_{0}+\ldots+\mathcal{M}_{q}\right|$.

With respect to the number of membranes of the system, by applying a separation rule for elementary membranes, an object is removed from the system, no new objects are produced and a new membrane is created. Thus, at most $M$ membranes can be produced by means of this process. Also, by applying a separation rule for non-elementary membranes, the number of objects remains unchanged but a new membrane is created (when such a rule is applied to a non-elementary membrane, it cannot be applied to that membrane anymore). In this way, no more than $q-2$ new membranes can be generated. Consequently, $q+M+(q-2)=M+2 q-2$ is an upper bound of the total number of membranes at $\mathcal{C}_{t}$.

- In order to identify the membranes created by the application of a separation rule, we modify the labels of the new membranes in the following recursive manner:

- The label of a membrane will be a pair $(i, \sigma)$ where $0 \leq i \leq q$ and $\sigma \in\{0,1\}^{*}$. At the initial configuration, the labels of the membranes are $(1, \lambda), \ldots,(q, \lambda)$. The label of the environment is denoted by $(0, \lambda)$.

- If a separation rule is applied to a membrane labeled by $(i, \sigma)$, then the new created membranes will be labeled by $(i, \sigma 0)$ and $(i, \sigma 1)$, respectively. Membrane $(i, \sigma 0)$ will only contain the objects of membrane $(i, \sigma)$ which belong to $\Gamma_{0}$, and membrane $(i, \sigma 1)$ will only contain the objects of membrane $(i, \sigma)$ which belong to $\Gamma_{1}$. Only elementary membranes can be separated, so if a membrane $i$ is non-elementary then we denote it by the label $(i, \lambda)$.

- If an object evolution rule or a communication rule is applied to a membrane labeled by $(i, \sigma)$, then after the application of the rule, the membrane keeps its label.

- Let us notice that the number of labels we need to identify all membranes appearing along any computation of a P system from $\mathcal{S A M}^{0}\left(+e_{s}, m_{c m p}\right.$ in-out $\left.,+d,+n\right)$ is of the order $O(M+q)$.

- A configuration $\mathcal{C}_{t}$ of a P system from $\mathcal{S} \mathcal{A} \mathcal{M}^{0}\left(+e_{s}, m c m p\right.$ in-out $\left.,+d,+n\right)$ is described by the current membrane structure and the multisets of labeled objects of the type

$$
\left\{(a, i, \sigma): a \in \Gamma, 0 \leq i \leq q, \sigma \in\{0,1\}^{*}\right\}
$$

The expression $(a, i, \sigma) \in \mathcal{C}_{t}$ means that object $a$ belongs to membrane labeled by $(i, \sigma)$.

- Let $r=[a \rightarrow b]_{h} \in \mathcal{R}$ be an object evolution rule of $\Pi$. We denote by $n \cdot L H S(r,(i, \sigma)), n \in \mathbf{N}$, the multiset of labeled objects $(a, i, \sigma)^{n}$ We denote by $n \cdot R H S(r,(i, \sigma))$ the multiset of labeled objects $(b, i, \sigma)^{n}$ produced by applying $n$ times rule $r$ over membrane $(i, \sigma)$.

- Let $r=[a b]_{h} \rightarrow c[]_{h} \in \mathcal{R}$ be a send-out communication rule of $\Pi$. We denote by $L H S(r,(i, \sigma))$ the labeled object $(a, i, \sigma)(b, i, \sigma)$. We denote by $R H S(r,(i, \sigma))$ the labeled object $(c, p(i), \tau)$ produced by applying rule $r$ over membrane $(i, \sigma)$, where $(p(i), \tau)$ is the parent of membrane $(i, \sigma)$. Similarly these concepts are defined for communication rules of the forms $[a]_{h} \rightarrow c[]_{h}$. 
- Let $r=a b[\quad]_{h} \rightarrow[c]_{h} \in \mathcal{R}$ be a send-in communication rule of $\Pi$. We denote by $\operatorname{LHS}(r,(i, \sigma))$ the labeled object $(a, p(i), \tau)(a, p(i), \tau)$, where $(p(i), \tau)$ is the parent of membrane $(i, \sigma)$. We denote by $R H S(r,(i, \sigma))$ the labeled object $(c, i, \sigma)$ produced by applying rule $r$ over membrane $(i, \sigma)$. Similarly these concepts are defined for communication rules of the forms $a[]_{h} \rightarrow[c]_{h}$.

- Let $\mathcal{C}_{t}$ be a configuration of $\Pi$, we denote by $\mathcal{C}_{t}+\left\{(x, i, \sigma) / \sigma^{\prime}\right\}$ the multiset obtained by replacing in $\mathcal{C}_{t}$ every occurrence of $(x, i, \sigma)$ by $\left(x, i, \sigma^{\prime}\right)$. Besides, $\mathcal{C}_{t}+m$ (resp., $\mathcal{C}_{t} \backslash m$ ) is used to denote that a multiset $m$ of labeled objects is added (resp., removed) to the configuration.

Next, we provide a deterministic algorithm $\mathcal{A}$ working in polynomial time that receives as input a recognizer $\mathrm{P}$ system $\Pi$ from $\mathcal{S} \mathcal{A M}^{0}\left(+e_{s}, m c m p\right.$ in-out $\left.,+d,+n\right)$ together with an input multiset $m$ of $\Pi$. Then algorithm $\mathcal{A}$ reproduces the behavior of a single computation of such system.

The pseudocode of the algorithm $\mathcal{A}$ is described as follows:

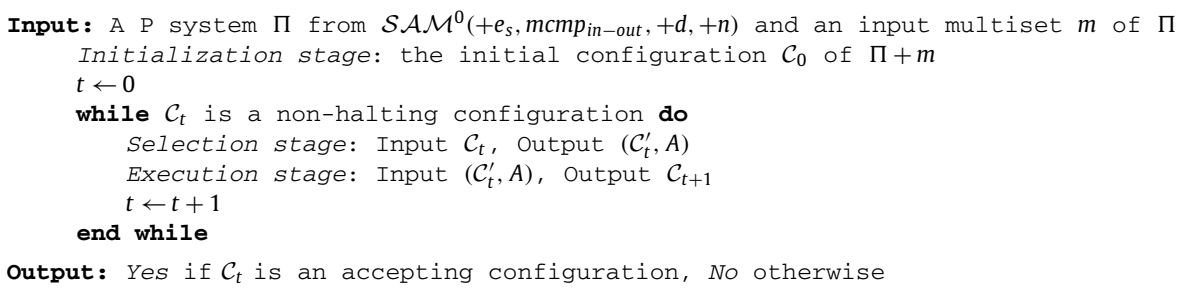

Output: Yes if $\mathcal{C}_{t}$ is an accepting configuration, No otherwise

The selection stage and the execution stage implement a transition step of a recognizer $\mathrm{P}$ system $\Pi$. Specifically, the selection stage receives as input a configuration $\mathcal{C}_{t}$ of $\Pi$ at an instant $t$. The output of this stage is a pair $\left(\mathcal{C}_{t}^{\prime}, A\right)$, where $A$ encodes a multiset of rules selected to be applied to $\mathcal{C}_{t}$, and $\mathcal{C}_{t}^{\prime}$ is the configuration obtained from $\mathcal{C}_{t}$ once the labeled objects corresponding to the application of rules from $A$ have been consumed. The execution stage receives as input the output $\left(\mathcal{C}_{t}^{\prime}, A\right)$ of the selection stage, and the output is the next configuration $\mathcal{C}_{t+1}$ of $\mathcal{C}_{t}$. Specifically, at this stage, configuration $\mathcal{C}_{t}^{\prime}$ yields configuration $\mathcal{C}_{t+1}$ by adding the labeled objects produced by the application of rules from $A$.

Next, selection stage and execution stage are described in detail.

\section{Selection stage.}

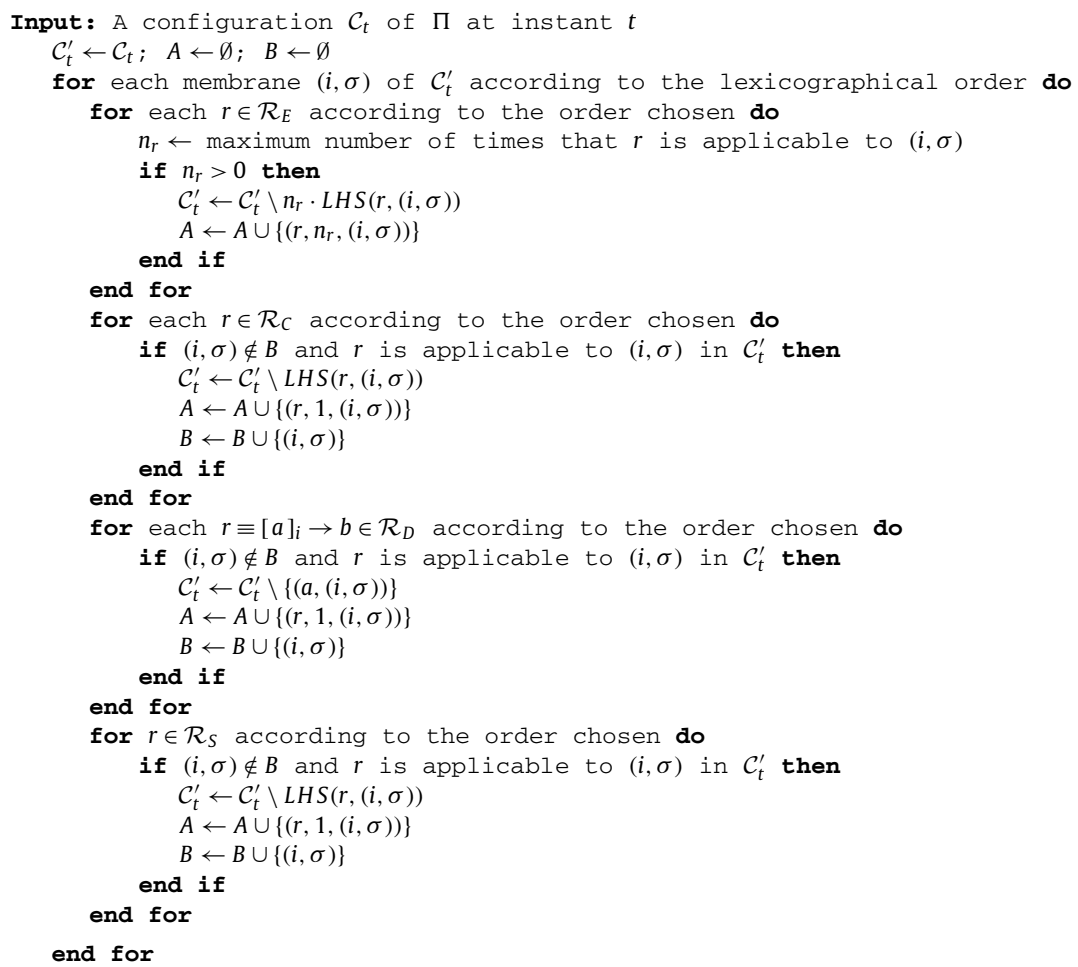

This algorithm is deterministic and works in polynomial time. Indeed, the cost in time is polynomial in the size of $\Pi$ because the number of cycles of the external main for loop is of order $O(M+q)$, and the number of cycles of the three internal main for loops are of order $O(|R|)$. Besides, the cost of each internal loop is of the order $O(M+q)$. 
Let us notice that the number of tuples in set $A$ is of the order $O(M)$ because each object in the system can be involved in, at most, one rule and at any configuration $\mathcal{C}_{t}$ the total number of objects is upper bounded by $M$. In set $A$ an order is considered in a natural way (a product order concerning the rules, natural numbers and labels).

In order to complete the simulation of a computation step of the system $\Pi$, the execution stage takes care of the effects of applying the rules selected in the previous stage: updating the objects according to the RHS of the rules.

\section{Execution stage.}

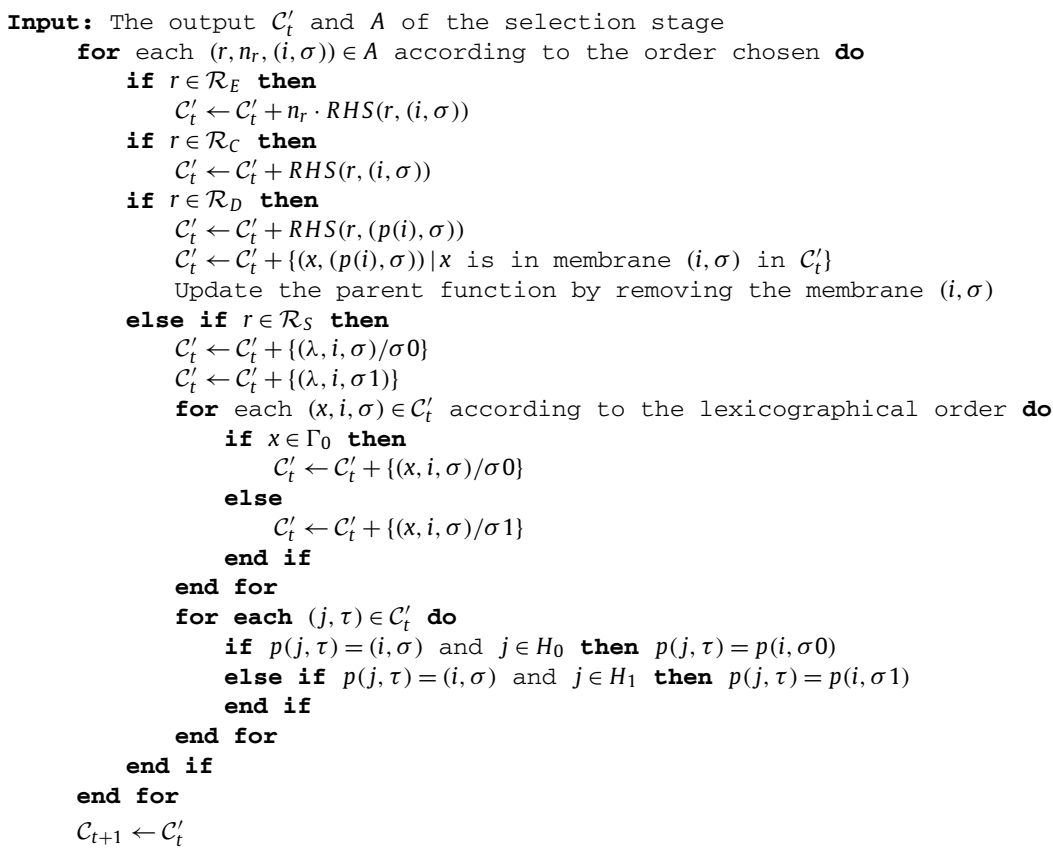

This algorithm is deterministic and works in polynomial time. Indeed, on the one hand, the number of cycles of the main for loop is of order $O(M)$. On the other hand, each cycle of the main for loop takes $O(|R|)$ steps plus the number of steps spent by the two secondary for loops: the first takes $O(M(M+q))$ steps and the second takes $O(M+q)$ steps.

Theorem 1. P $=$ PMC $_{\mathcal{S} \mathcal{A M}}{ }_{\left(+e_{s}, m c m p\right.}$ in-out $\left.,+d,+n\right)$.

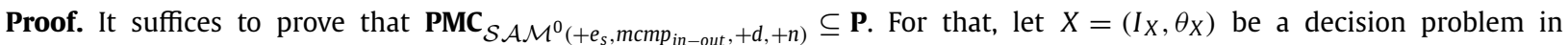
PMC $_{\mathcal{S A M}^{0}\left(+e_{s}, m c m p_{\text {in-out }},+d,+n\right)}$. Let $\{\Pi(n) \mid n \in \mathbb{N}\}$ be a family of P systems from $\mathcal{S A M}^{0}\left(+e_{s}, m c m p_{\text {in-out }},+d,+n\right)$ solving $X$, according to Definition 4.1 . Let $(\operatorname{cod}, s)$ be a polynomial encoding associated with that solution. Let us recall that instance $u \in I_{X}$ of the problem $X$ is processed by the system $\Pi(s(u))+\operatorname{cod}(u)$.

Let us consider the following deterministic algorithm $\mathcal{A}^{\prime}$ :

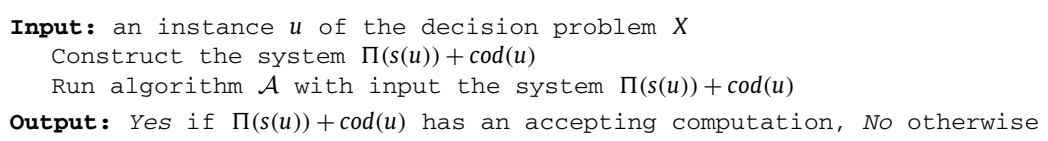

Given an instance $u$ of the decision problem $X=\left(I_{X}, \theta_{X}\right)$, the following assertions are equivalent:

1. $\theta_{X}(u)=1$, that is, the answer of problem $X$ to instance $u$ is affirmative.

2. Every computation of $\Pi(s(u))+\operatorname{cod}(u)$ is an accepting computation.

3. The output of the algorithm with input $u$ is Yes.

Therefore, algorithm $\mathcal{A}^{\prime}$ provides a solution of the decision problem $X$. Bearing in mind that $\mathcal{A}^{\prime}$ works in polynomial time, we finally deduce that $X \in \mathbf{P}$.

\section{Simple object evolution rules}

In [1] a polynomial-time solution to QSAT, a well-known PSPACE-complete problem is given in terms of polarizationless $P$ systems with active membranes that make use of division rules for both elementary and non-elementary membranes and 
dissolution rules. We can provide a tighter bound, by using only simple object evolution rules, that is, only one object is used in the RHS of this kind of rules. In order to obtain this solution, we are going to describe briefly the changes needed.

Theorem 2. QSAT $\in$ PMC $_{\mathcal{D} \mathcal{A} \mathcal{M}^{0}\left(+e_{s},+c,+d,+n\right)}$

Let $\Pi(\langle n, p\rangle)=\left(\Gamma, H, \mu, \mathcal{M}_{0}, \ldots, \mathcal{M}_{m+5 n+3}, \mathcal{R}\right)$ be the family of P systems defined in [1]. Then, we define:

$$
\Pi^{\prime}(\langle n, p\rangle)=\left(\Gamma^{\prime}, H^{\prime}, \mu^{\prime}, \mathcal{M}_{0}^{\prime}, \ldots, \mathcal{M}_{m+5 n+3}^{\prime}, \mathcal{R}^{\prime}\right),
$$

where:

(1) $\Gamma^{\prime}=\Gamma^{-} \cup\left\{a_{i, j} \mid 1 \leq i \leq n, 0 \leq j \leq 3 n-2\right\}$, being $\Gamma^{-}=\Gamma \backslash\left\{a_{i} \mid 1 \leq i \leq n\right\}$;

(2) $H^{\prime}=H$;

(3) $\mu^{\prime}=\mu$;

(4) $\mathcal{M}_{0}^{\prime}=\mathcal{M}_{0} \cup\left\{a_{i, 0} \mid 0 \leq i \leq n-1\right\}, \mathcal{M}_{k}^{\prime}=\mathcal{M}_{k}, 1 \leq k \leq m+5 n+3$;

(5) $\mathcal{R}^{\prime}=\mathcal{R} \backslash\{G 1, G 2\} \cup\left\{G 1^{\prime}, G 2^{\prime}\right\}$

$$
\begin{aligned}
G 1^{\prime} & {\left[a_{i, 3 j} \rightarrow a_{i, 3 j+1}\right]_{0} \text { for } 1 \leq i \leq n, 0 \leq j \leq i-1 } \\
& {\left[a_{i, 3 j+1} \rightarrow a_{i, 3 j+2}\right]_{0} } \\
& {\left.\left[a_{i, 3 j+2} \rightarrow a_{i, 3 j+3}\right]_{0}\right\} \text { for } 1 \leq i \leq n, 0 \leq j \leq i-2 } \\
& {\left[d_{i} \rightarrow d_{i+1}\right]_{0} \text { for } 0 \leq i<5 n+p }
\end{aligned}
$$

We count to $5 n+p$ with the $d_{i}$ object, same as in the reference, but this object is not concerned over the division nor creating new objects $a_{i}$. Instead of it, objects $a_{i, j}$ are in the initial multisets and are the only ones in charge of the division of membranes thank to the rules of $\mathrm{G} 2$.

$G 2^{\prime}\left[a_{i, 3 i-2}\right]_{0} \rightarrow\left[t_{i}\right]_{0}\left[f_{i}\right]_{0}$ for $1 \leq i \leq n$

In this model, membranes divide at the exact same step than in the original one, so it is easy to see that the change of these rules only affects the generation stage, so the rest of the computation keeps the same behavior as in the original design.

Corollary 1. PSPACE $=\mathbf{P M C}_{\mathcal{D} \mathcal{A} \mathcal{M}^{0}\left(+e_{s},+c,+d,+n\right)}$

Proof. On the one hand, it is easy to prove that

PSPACE $\subseteq$ PMC $_{\mathcal{D A} \mathcal{A} \mathcal{M}^{0}\left(+e_{s},+c,+d,+n\right)}$

from Theorem 2 and taking account that class $\mathbf{P M C}_{\mathcal{D A M}} \mathcal{A M}^{0}\left(+e_{s},+c,+d,+n\right)$ is closed under polynomial time reductions.

On the other hand, we only have to remember that

$$
\text { PMC }_{\mathcal{D} \mathcal{A M}}^{0}\left(+e_{s},+c,+d,+n\right) \subseteq \text { PMC }_{\mathcal{D} \mathcal{A M}}(+e,+c,+d,+n) \subseteq \text { PSPACE. }
$$

In [18], it is demonstrated that efficiency cannot be reached using minimal cooperation with polarizationless $\mathrm{P}$ systems with active membranes and separation rules, even if dissolution and separation for both elementary and non-elementary membranes are allowed. From this result, we can extract some information not only about polarizationless P systems with active membranes which use separation rules and simple object evolution rules, but with these systems using polarizations, we can keep using the algorithmic technique in order to simulate them, since polarizations do not add any object to the system, so the system workspace remains upper bounded by a polynomial function.

$$
\text { Corollary 2. } \mathbf{P}=\mathbf{P M C}_{\mathcal{S} \mathcal{A M}\left(+e_{s},+c,+d,+n\right)}
$$

\section{Upper bounds of cooperation}

A new frontier of the efficiency has been obtained in the framework of polarizationless P systems with active membranes which make use of minimal cooperation rules in communication rules. On the one hand, from the previous section we have obtained that systems from $\mathcal{S} \mathcal{A} \mathcal{M}^{0}\left(+e_{s}, m c m p\right.$ in-out $\left.,+d,+n\right)$ can only solve problems from the class $\mathbf{P}$, obtaining a landscape of these systems as shown in the below figure of Fig. 6.

On the other hand, in [22,23] a uniform polynomial time solution to SAT problem by families of polarizationless P systems with active membranes and with division rules which make use of minimal cooperation and minimal production in send-in (resp., send-out) communication rules have been provided.

Corollary 3. NP $\cup$ co $-\mathbf{N P} \subseteq \mathbf{P M C}_{\mathcal{D} \mathcal{A} \mathcal{M}^{0}\left(+e_{s}, m c m p_{\text {in }},-d,+n\right)} \cap \mathbf{P M C}_{\mathcal{D} \mathcal{A} \mathcal{M}^{0}\left(+e_{s}, m c m p_{\text {out }},-d,+n\right)}$ 


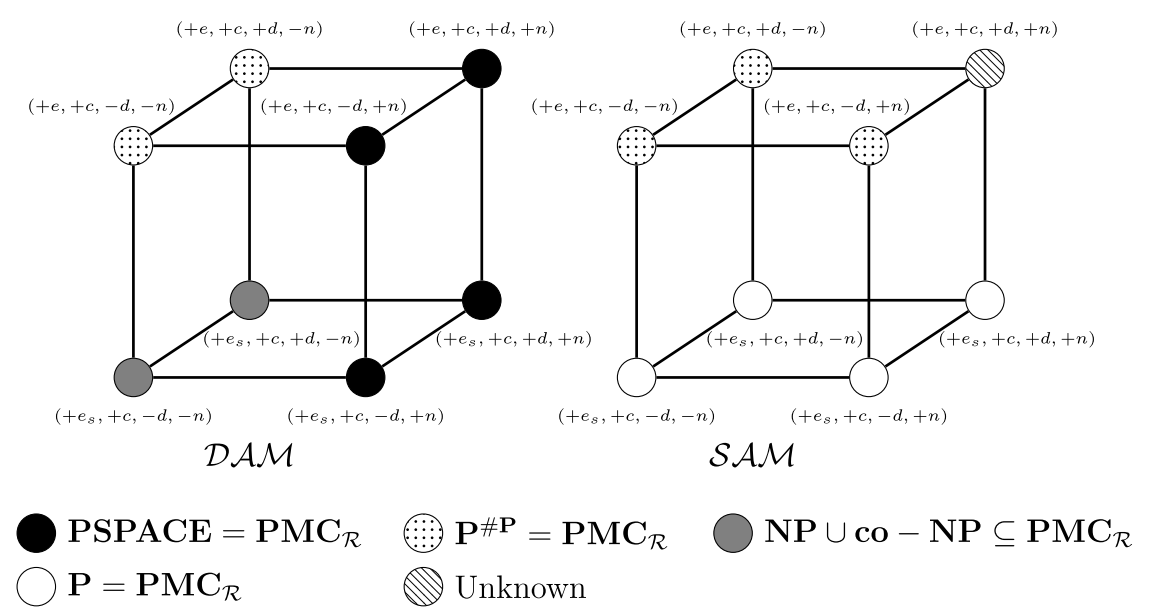

Fig. 1. Efficiency in P systems with active membranes.

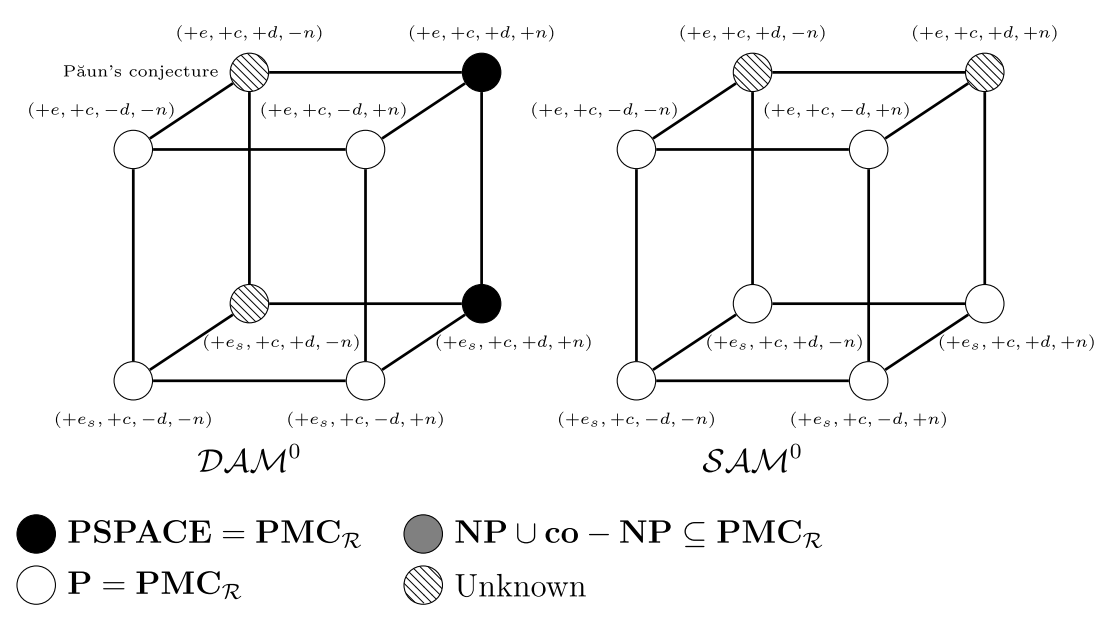

Fig. 2. Efficiency in polarizationless $P$ systems with active membranes.

Apart from that, and using the same technique from [18], that is, taking the solution from [21] and generating all the objects using object evolution rules of the type $[a \rightarrow b c]_{h}$ in order to obtain an exponential workspace with regard of the objects present in the system. In this case, if we take the solution of [22,23] and apply this technique, the following holds:

Corollary 4. $\mathbf{N P} \cup \mathbf{c o}-\mathbf{N P} \subseteq \mathbf{P M C}_{\mathcal{S A M}^{0}\left(+e, m c m p_{i n},-d,+n\right)} \cap \mathbf{P M C}_{\mathcal{S A M}^{0}{ }^{0}\left(+e, m c m p_{\text {out }},-d,+n\right)}$,

so below figure of Fig. 5 differs from the one from Fig. 6.

It gives us a new frontier in polarizationless $P$ systems with active membranes which make use of separation rules and minimal cooperation in communication rules with respect of the size of object evolution rules.

Techniques given in $[16,17]$ can be easily adapted to the systems studied in this work and new characterizations by means of classical complexity classes can be obtained.

From classical results in [5] to new ones obtained in this work, a state-of-art representation of complexity classes regarding cooperation in P systems with active membranes is contained in Figs. 1, 2, 3, 4, 5 and 6.

\section{Conclusions and open problems}

The classical definition of polarizationless $\mathrm{P}$ systems with active membranes makes use of non-cooperative rules and their object evolution rules are of the form $[a \rightarrow u]_{h}$, where $a$ is an object of the working alphabet and $u$ is a finite multiset of objects. In that context, the capability of these membrane systems to create an exponential workspace in polynomial time is implemented by means of division rules (for both elementary and non-elementary membranes). It is well known [3] that only tractable problems can be solved in an efficient way by families of such kind of P systems which do not make use of dissolution rules even if division rules for elementary and non-elementary membranes is allowed, that is, 


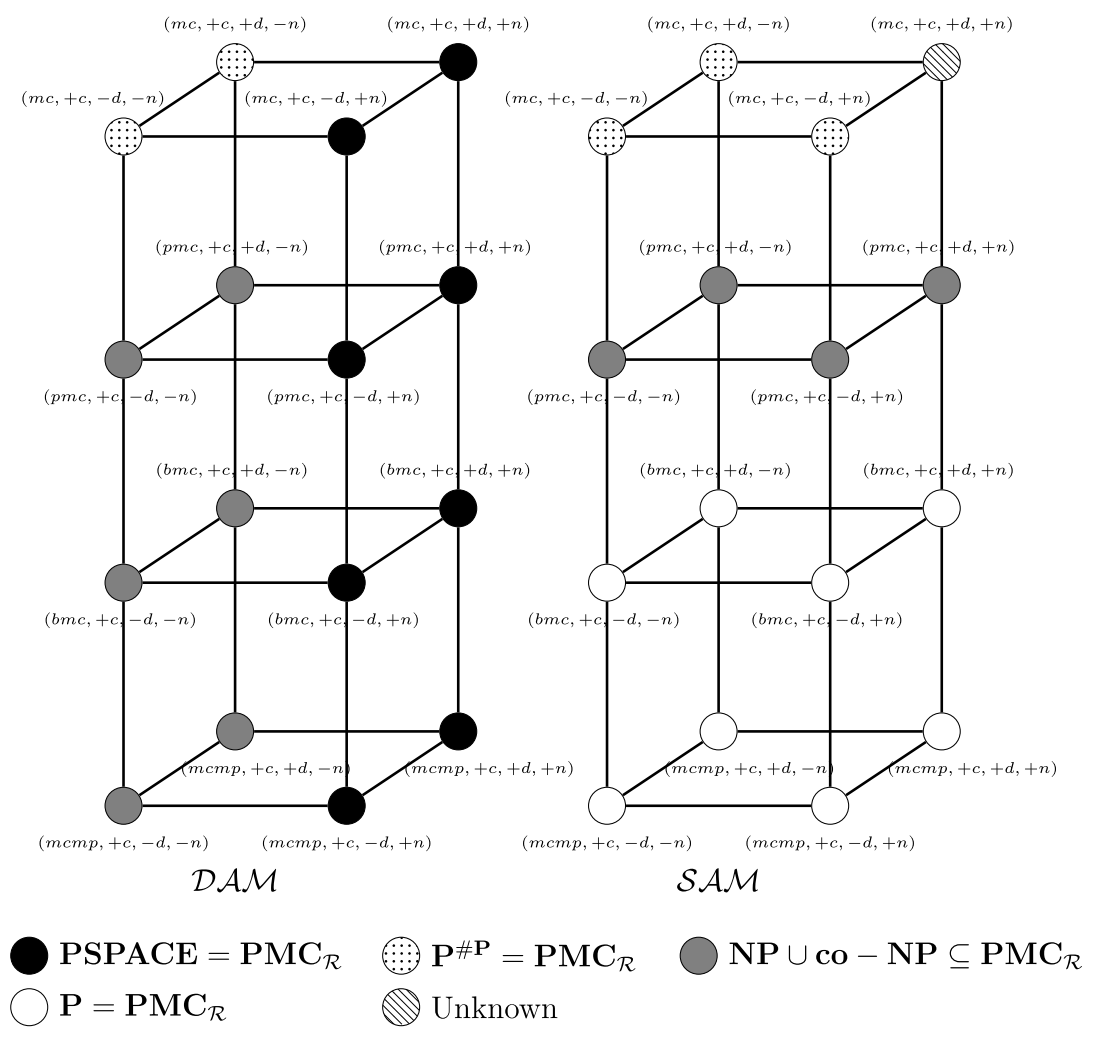

Fig. 3. Efficiency in P systems with active membranes which make use of cooperation in object evolution rules.

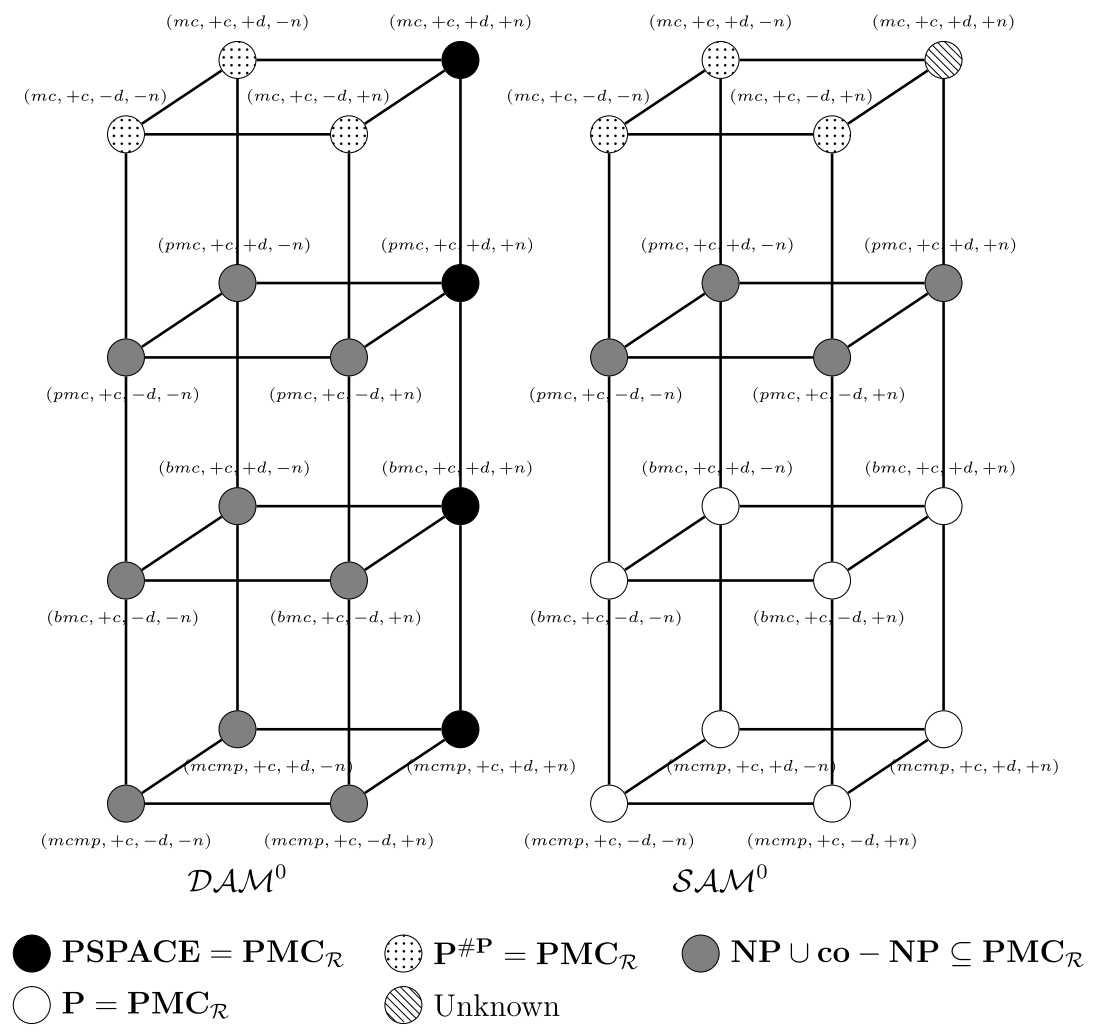

Fig. 4. Efficiency in polarizationless $P$ systems with active membranes which make cooperation in object evolution rules. 

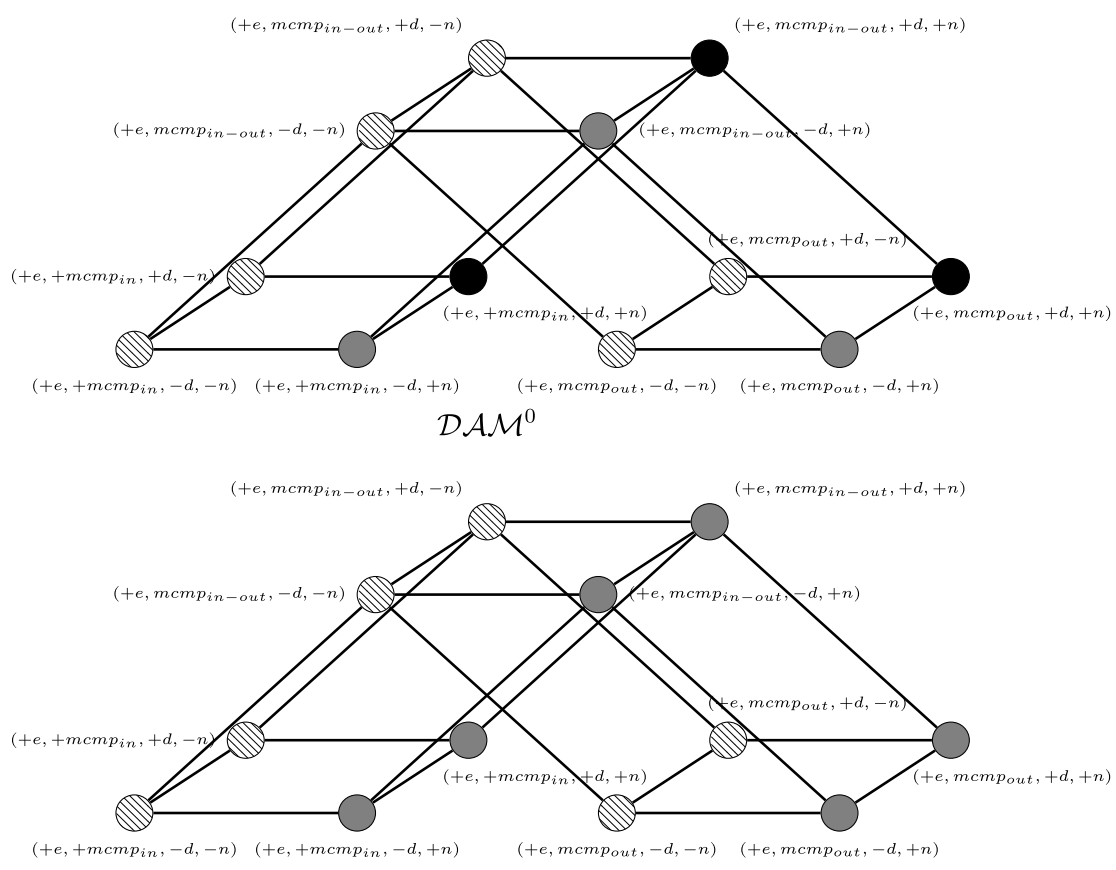

$\mathcal{S A M} \mathcal{M}^{0}$

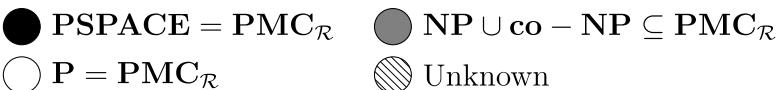

Fig. 5. Efficiency in polarizationless $P$ systems with active membranes which make use of cooperation in communication rules.

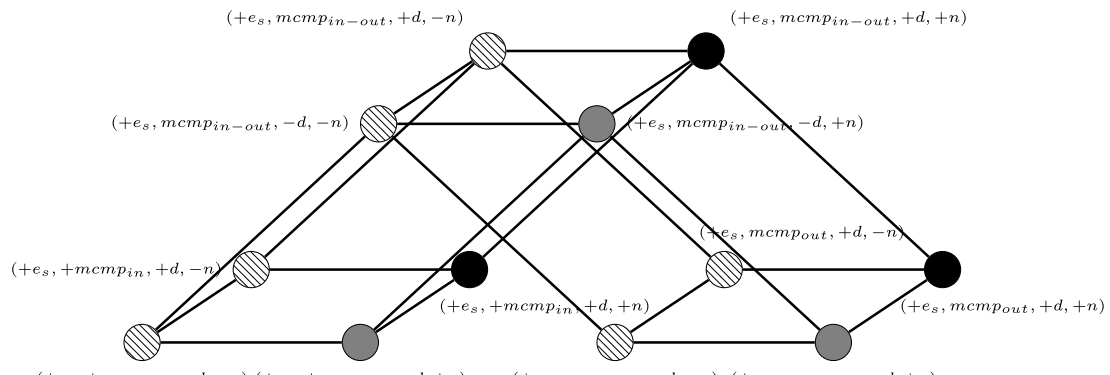

$\left(+e_{s},+m c m p_{i n},-d,-n\right)\left(+e_{s},+m c m p_{i n},-d,+n\right) \quad\left(+e_{s}, m c m p_{\text {out }},-d,-n\right) \quad\left(+e_{s}, m c m p_{\text {out }},-d,+n\right)$

$\mathcal{D} \mathcal{A} \mathcal{M}^{0}$

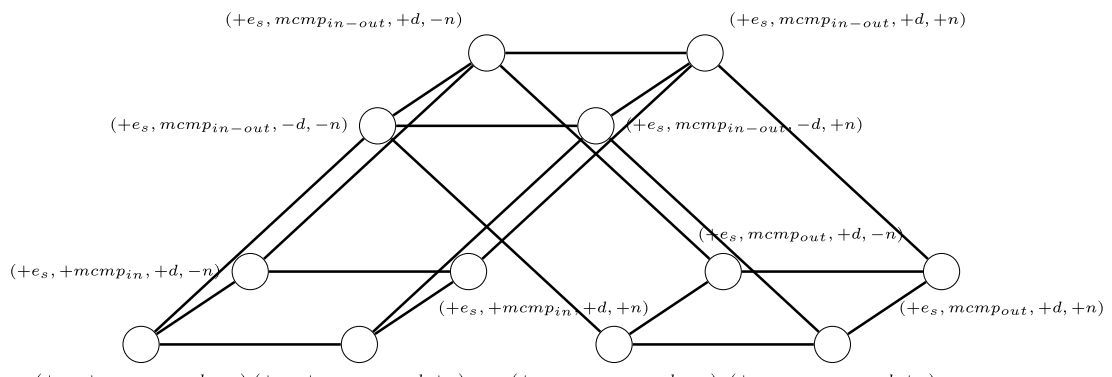

$\left(+e_{s},+m c m p_{i n},-d,-n\right)\left(+e_{s},+m c m p_{i n},-d,+n\right) \quad\left(+e_{s}, m c m p_{\text {out }},-d,-n\right)\left(+e_{s}, m c m p_{\text {out }},-d,+n\right)$

$\mathcal{S A M} \mathcal{M}^{0}$

PSPACE $=$ PMC $_{\mathcal{R}}$

$\mathbf{P}=\mathbf{P M C}_{\mathcal{R}}$

$\mathrm{NP} \cup$ co $-\mathrm{NP} \subseteq \mathrm{PMC}_{\mathcal{R}}$

Unknown

Fig. 6. Efficiency in polarizationless $P$ systems with active membranes which make use simple evolution and cooperation in communication rules. 
$\mathbf{P}=\mathbf{P M C}_{\mathcal{D A M}^{0}(+e,+c,-d,+n)}$. Besides, in this context dissolution rules play an important role because of this kind of rules are permitted then PSPACE-complete problems can be solved efficiently [1], that is PSPACE $\subseteq$ PMC $_{\mathcal{D A M}}{ }^{0}(+e,+c,+d,+n)$.

In this paper, we compare these systems with their cooperative counterparts, using both division and separation and using cooperation in both object evolution and communication rules.

In Section 7 we study the capabilities of polarizationless P systems with active membranes that make use of separation rules and minimal cooperation in communication rules, both in send-in and send-out. Using simple evolution rules, that is, only one object in the right-hand side of the rule, we obtain that this kind of systems can only solve problems from class $\mathbf{P}$, obtaining a new frontier of efficiency regarding the kind of evolution rules, since if we use classical evolution rules $[a \rightarrow u]_{h}$, we can solve problems from class NP, adapting the solutions from [22,23].

In the next section, we improve the results from [1], using simple evolution rules instead of classical ones, and yet obtaining enough computational power to solve problems from PSPACE in polynomial time. It gives us a hint that using division rules leaves the length of the right-hand side of object evolution rules in the background.

Taking a look at the figures, we can see that when we use separation rules and simple object evolution rules, even using polarizations or bounded minimal cooperation is not enough to reach efficiency. But using separation rules besides object evolution rules allowing the RHS to have a greater length than the LHS allows the system have the same efficiency as their division counterparts, because we can simulate the behavior of division just by separating and then duplicating the objects of the two new membranes, that is, by allowing the system generate an exponential workspace with regard of the number of objects (let us remind that if a system uses separation rules and simple evolution rules, the number of objects and membranes comes bounded by the initial number of them, so it cannot create an unlimited exponential workspace as membrane systems using division).

Another conclusion we can make is that the upper bound of efficiency of membrane systems remains at PSPACE, because we can adapt easily the algorithm defined in [13] to membrane systems using cooperation.

Some open problems are the next ones:

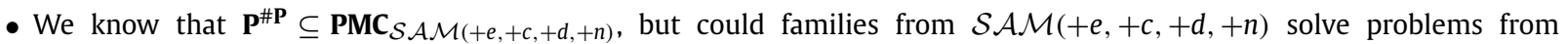
PSPACE in polynomial time?

- The Păun conjecture remains open also for P systems from $\mathcal{D} \mathcal{A} \mathcal{M}^{0}\left(+e_{s},+c,+d,-n\right)$ and $\mathcal{S} \mathcal{A} \mathcal{M}^{0}(+e,+c,+d,-n)$.

- Can systems from $\mathcal{S} \mathcal{A} \mathcal{M}^{0}(+e,+c,+d,+n)$ reach efficiency?

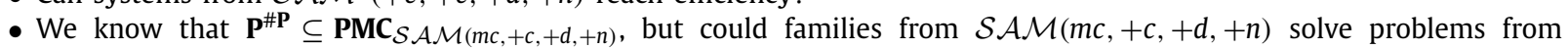
PSPACE in polynomial time?

- We know that $\mathbf{P}^{\# \mathbf{P}} \subseteq \mathbf{P M C}_{\mathcal{S} \mathcal{A} \mathcal{M}^{0}(m c,+c,+d,+n)}$, but could families from $\mathcal{S A M}^{0}(m c,+c,+d,+n)$ solve problems from PSPACE in polynomial time?

- We do not know if division rules for non-elementary membranes are a requisite to reach efficiency while using cooperation in communication rules.

\section{Acknowledgements}

This work was partially supported by Project TIN2017-89842-P of the Ministerio de Economía y Competitividad of Spain and by Grant number 61320106005 of the National Natural Science Foundation of China.

\section{References}

[1] A. Alhazov, M.J. Pérez-Jiménez, Uniform solution of QSAT using polarizationless active membranes, in: Lecture Notes in Computer Science, vol. 4664, 2007, pp. 122-133.

[2] T.H. Cormen, C.E. Leiserson, R.L. Rivest, An Introduction to Algorithms, The MIT Press, Cambridge, Massachusetts, 1994.

[3] M.A. Gutiérrez-Naranjo, M.J. Pérez-Jiménez, A. Riscos-Núñez, F.J. Romero-Campero, On the Power of Dissolution in P Systems with Active Membranes, Springer, Berlin, Heidelberg, 2006, pp. 224-240.

[4] M.A. Gutiérrez-Naranjo, M.J. Pérez-Jiménez, A. Riscos-Núñez, F.J. Romero-Campero, A. Romero-Jiménez, Characterizing tractability by cell-like membrane systems, in: K.G. Subramanian, K. Rangarajan, M. Mukund (Eds.), Formal Models, Languages and Applications, World Scientific, Singapore, 2006, pp. 137-154.

[5] Gh. Păun, Attacking NP-complete problems, in: I. Antoniou, C. Calude, M.J. Dinneen (Eds.), Unconventional Models of Computation, UMC'2K, SpringerVerlag, 2000, pp. 94-115.

[6] Gh. Păun, P systems with active membranes: attacking NP-complete problems, J. Autom. Lang. Comb. 6 (1) (2001) 75-90.

[7] Gh. Păun, G. Rozenberg, A. Salomaa (Eds.), The Oxford Handbook of Membrane Computing, Oxford University Press, Oxford, 2010.

[8] M.J. Pérez-Jiménez, An approach to computational complexity in Membrane Computing, in: G. Mauri, Gh. Păun, M.J. Pérez-Jiménez, G. Rozenberg, A. Salomaa (Eds.), Membrane Computing, International Workshop, WMC5, Selected Papers, Milano, Italy, 2004, in: Lecture Notes in Computer Science, vol. 3365, 2005, pp. 85-109.

[9] M.J. Pérez-Jiménez, A. Romero-Jiménez, F. Sancho-Caparrini, Complexity classes in cellular computing with membranes, Nat. Comput. 2 (3) (2003) 265-285.

[10] M.J. Pérez-Jiménez, A. Romero-Jiménez, F. Sancho-Caparrini, A polynomial complexity class in P systems using membrane division, J. Autom. Lang. Comb. 11 (4) (2006) 423-434. A preliminary version in E. Csuhaj-Varjú, C. Kintala, D. Wotschke, Gy. Vaszil (Eds.), Proceedings of the Fifth International Workshop on Descriptional Complexity of Formal Systems, DCFS 2003, Budapest, Hungary, July 12-14, 2003, pp. 284-294.

[11] M.J. Pérez-Jiménez, A computational complexity theory in membrane computing (invited talk) in: Membrane Computing, 10th International Workshop, WMC 2009, Revised Selected and Invited Papers, Curtea de Arges, Romania, August 24-27, 2009, in: Lecture Notes in Computer Science, vol. 5957, 2010, pp. 125-148. 
[12] A.E. Porreca, G. Mauri, C. Zandron, Complexity classes for membrane systems, Inform. Théor. Appl. 40 (2) (2006) $141-162$.

[13] P. Sosík, A. Rodríguez-Patón, Membrane computing and complexity theory: a characterization of PSPACE, J. Comput. System Sci. 73 (2007) 137-152.

[14] L. Pan, T.-O. Ishdorj, P systems with active membranes and separation rules, J. Univers. Comput. Sci. 10 (5) (2004) 630-649.

[15] Gh. Păun, Computing with membranes, J. Comput. Syst. Sci. Int. 61 (1) (2000) 108-143.

[16] A.E. Porreca, A. Leporati, G. Mauri, C. Zandron, P systems simulating oracle computations, in: Membrane Computing: 12th International Conference, CMC 2011, vol. 7184, Fontainebleau, France, August 23-26, 2011, 2012, pp. 346-358.

[17] A. Leporati, L. Manzoni, A.E. Porreca, C. Zandron, Simulating elementary active membranes with an application to the P conjecture, in: Membrane Computing: 15th International Conference, CMC 2014, vol. 8961, Prague, Czech Republic, August 20-22, 2014, pp. 284-299.

[18] L. Valencia-Cabrera, D. Orellana-Martín, M.Á. Martínez-del-Amor, A. Riscos-Núñez, M.J. Pérez-Jiménez, Computational efficiency of minimal cooperation and distribution in polarizationless P systems with active membranes, Fund. Inform. 153 (1-2) (2017) $147-172$.

[19] L. Valencia-Cabrera, D. Orellana-Martín, M.Á. Martínez-del-Amor, A. Riscos-Núñez, M.J. Pérez-Jiménez, From distribution to replication in cooperative systems with active membranes: a frontier of the efficiency, Theoret. Comput. Sci. 736 (2018) 15-24.

[20] L. Valencia-Cabrera, D. Orellana-Martín, M.Á. Martínez-del-Amor, A. Riscos-Núñez, M.J. Pérez-Jiménez, Minimal cooperation in polarizationless P systems with active membranes, in: Proceedings of the Fourteenth Brainstorming Week on Membrane Computing, Seville, Spain, February 1-5, 2016, Fénix Editora, 2017, pp. 327-356.

[21] L. Valencia-Cabrera, D. Orellana-Martín, M.Á. Martínez-del-Amor, A. Riscos-Núñez, M.J. Pérez-Jiménez, Reaching efficiency through collaboration in membrane systems: dissolution, polarization and cooperation, Theoret. Comput. Sci. 701 (2017) 226-234.

[22] L. Valencia-Cabrera, D. Orellana-Martín, M.Á. Martínez-del-Amor, A. Riscos-Núñez, M.J. Pérez-Jiménez, Restricted polarizationless P systems with active membranes: minimal cooperation only inwards, in: Proceedings of the Fifteenth Brainstorming Week on Membrane Computing, Report RGNC 1/2017, Seville, Spain, January 31-February 3, 2017, Fénix Editora, 2017, pp. 215-252.

[23] L. Valencia-Cabrera, D. Orellana-Martín, M.Á. Martínez-del-Amor, A. Riscos-Núñez, M.J. Pérez-Jiménez, Restricted polarizationless P systems with active membranes: minimal cooperation only outwards, in: Proceedings of the Fifteenth Brainstorming Week on Membrane Computing, Report RGNC 1/2017, Seville, Spain, January 31-February 3, 2017, Fénix Editora, 2017, pp. 253-290. 